\title{
Aortic Valve Replacement in Bicuspid Aortic Valve with a Single Coronary Artery
}

\author{
Masashi Kawabori \\ Division of Cardiac Surgery, Brigham and Women's Hospital, Harvard Medical School, Boston, MA, USA \\ Email: kawabori.masashi@gmail.com
}

Received 28 October 2015; accepted 11 December 2015; published 14 December 2015

Copyright @ 2015 by author and Scientific Research Publishing Inc.

This work is licensed under the Creative Commons Attribution International License (CC BY).

http://creativecommons.org/licenses/by/4.0/

c) (i) Open Access

\begin{abstract}
Coronary artery anomalies combined with bicuspid aortic valve are rare but cause perioperative complications if not detected on time. Because anomalous coronary arteries vary with respect to the ostium's origin and transverse route, the precise anatomy should be identified preoperatively and the procedure modified. We describe a severely stenotic bicuspid aortic valve and anomalous coronary artery corrected successfully with a modified valve replacement procedure, illustrating the importance of analyzing coronary anatomy preoperatively, regardless of age, to avoid occluding an anomalous artery's ostium and to address the anomalies. We describe our modified procedure based on the artery's location for a safe operation.
\end{abstract}

\section{Keywords}

Aortic Valve Stenosis, Bicuspid Aortic Valve, Coronary Angiography, Coronary Vessels, Heart Defects, Congenital

\section{Background}

An anomalous coronary artery, including a single coronary artery, usually has an anomalous origin and traverses atypically. Anomalies are found in $1 \%-2 \%$ of patients undergoing cardiac catheterization [1] [2]. Lipton and colleagues proposed three groups and nine subgroups for categorizing single coronary artery anomalies depending on the site of origin and the main branches' anatomical distribution [3]. Though rare, anomalous coronary arteries can lead to serious complications during cardiac surgery if not recognized preoperatively. We describe such an anomaly identified before elective replacement of a bicuspid aortic valve.

\section{Case Presentation}

A 57-year-old man (body surface area, $1.9 \mathrm{~m}^{2}$ ) presented with exertional dyspnea. Transthoracic echocardio- 
graphy revealed a calcified bicuspid aortic valve (Figure 1) with a mean pressure gradient of $63 \mathrm{mmHg}$ and a calculated aortic valve area of $0.78 \mathrm{~cm}^{2}$, consistent with severe stenosis. Left ventricular ejection fraction was preserved at $65 \%$. Preoperative coronary angiography demonstrated a single coronary artery above the right coronary cusp. The left coronary artery originated from the proximal portion of the right coronary artery (Figure 2). Three-dimensional computed tomography angiography demonstrated that the left coronary artery traveled anteriorly from its anomalous origin and across the conus arteriosus before anatomically correct bifurcation into left anterior descending and left circumflex vessels (Figure 3). No associated coronary artery disease was observed. Aortic valve replacement was performed via full median sternotomy. The ascending aorta was cannulated, and venous cannulation was performed through the right atrium. The patient's core body temperature was slowly decreased to $35.5^{\circ} \mathrm{C}$. Myocardial protection was achieved using a combination of antegrade and retrograde cold bloodcardioplegia. The annulus sized to a 23 valve. The right coronary artery ostium was identified above the right coronary cusp via preoperative three-dimensional computed tomography angiography. The right coronary artery ostium was only 7 mmabove the annulus. We modified the procedure because of this proximity and the associated risk of coronary ostium occlusion if we set the valve supra-annularly. The 21-mm Aortic Magna Ease Valve (Edwards, Inc., Irvine, CA) was placed intra-annularly using everting sutures. The patient recovered uneventfully and was discharged home on postoperative day 7 . He provided written informed consent for publication of this case report and all accompanying images.

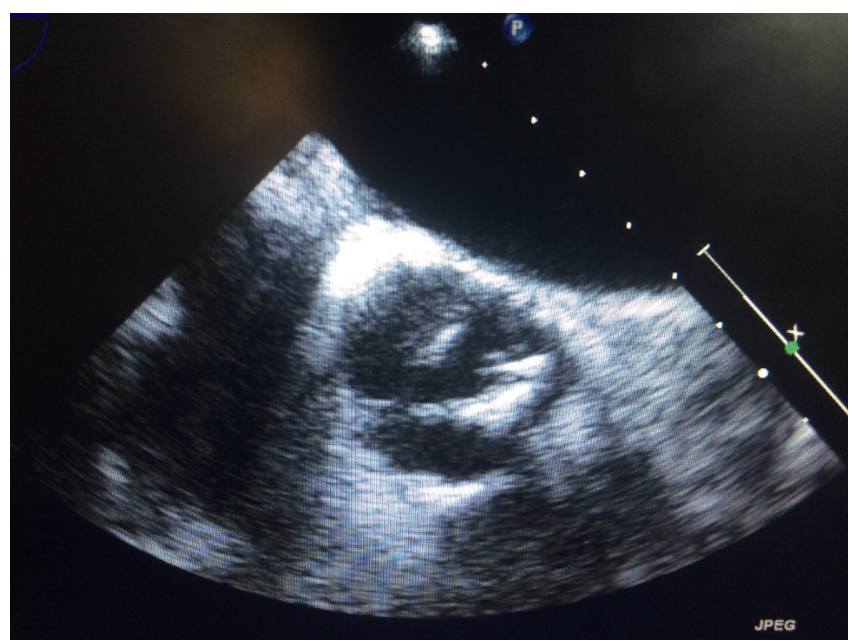

Figure 1. Severely calcified bicuspid aortic valve.

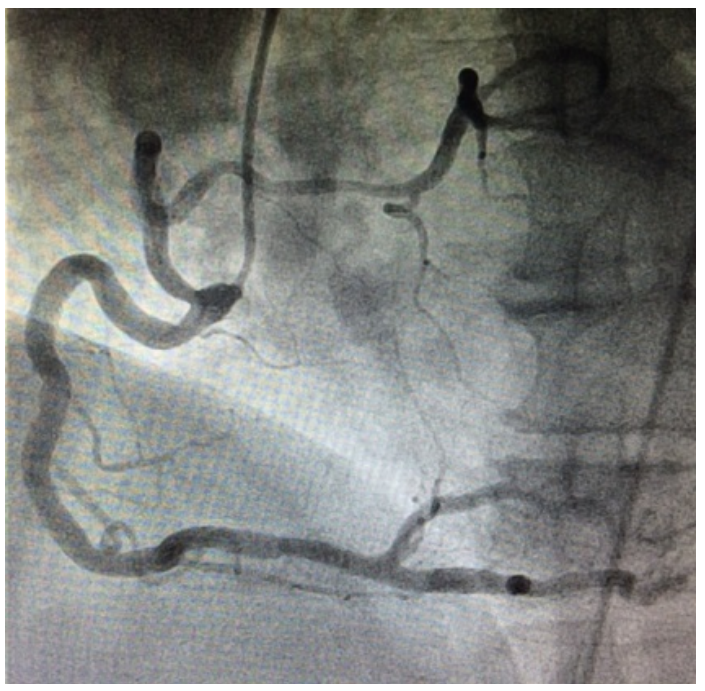

Figure 2. Single coronary angiography. 


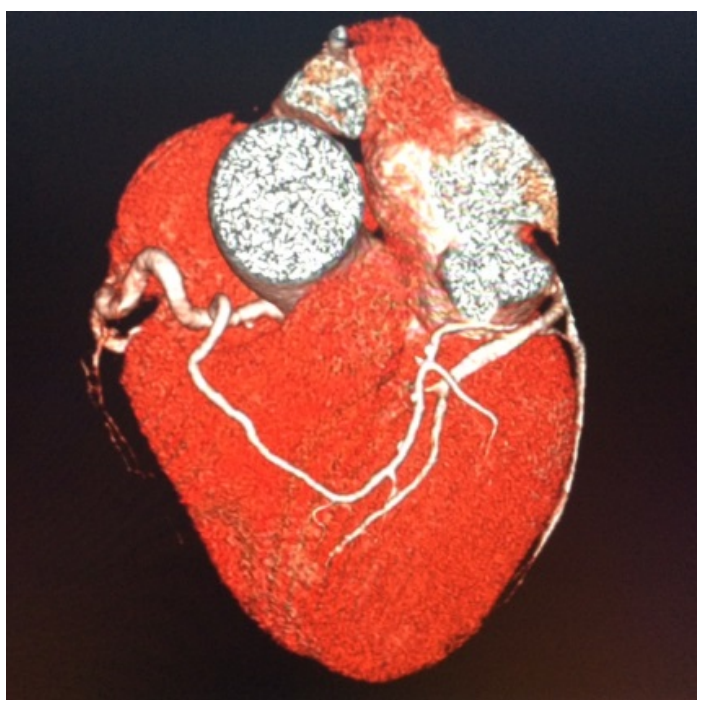

Figure 3. Single coronary 3D CT angiography.

\section{Discussion}

In this case, a single anomalous coronary artery was classified as Group II, Type R II-A because the left anterior descending artery arose from the right coronary artery and traversed the anterior surface to reach the inter ventricular groove [3]. As our patient had the anomalous coronary artery and bicuspid aortic valve, many individuals with a single coronary artery have additional congenital cardiac anomalies. Ogden and colleagues reported that 56/142 patients with a single coronary artery had other congenital cardiac anomalies; seven also had a bicuspid aortic valve [4].

Preoperative angiographic analysis of coronary anatomy is of paramount importance for preventing inadvertent damage to aberrantly located vessels during the operation. $\mathrm{T}$ here have been several reports of patients requiring aortic valve replacement for whom the anomalous circumflex coronary artery was compressed by the prosthetic valve's sewing ring's annulus [5]. Postoperative myocardial infarction and sudden death several years postoperatively have been reported [6]. Valveundersizing is a technique suggested to avoid undue compression of an anomalous coronary artery [7] [8]. Although our patient had a 23-mm annulus, a 21-mm valve was placed to avoid potential complications. Additionally, during surgery, we found that the coronary ostium was close to the annulus and that the valve prosthesis would sit on top of the coronary ostium if placed supra-annularly; therefore, we modified the procedure for setting the valve. We used an intra-annular technique instead of a supra-annular technique to avoid possible coronary ostium obstruction by the valve itself. This procedure did not result in a prosthetic mismatch. We also used retrograde cardioplegia for additional myocardial protection during the ischemic period.

Coronary anomalies can be readily identified in elderly patients with valvular disease, for whom cardiac catheterization is the standard of care; however, younger patients without risk factors for ischemic heart disease may not undergo preoperative coronary anatomy assessment. Given the potential for unrecognized coronary anomalies, it appears reasonable to consider coronary imaging for any patient undergoing cardiac surgery evaluation. In younger patients with few or no signs of ischemic heart disease, computed tomography angiography may be less invasive than is catheterization.

\section{Conclusion}

Our case illustrates the importance of analyzing coronary anatomy prior to valve surgery regardless of the patient's age to avoid damaging the anomalous arteries and to address the anomalies during the operation. Depending on the configuration of the coronary ostium and artery, surgeons should consider modifying the valve size selection method and the valve-setting procedure. Retrograde cardioplegia is as effective in this single coronary case as it is in normal cases. Because of the late complication risks related to these anomalies, postoperative imaging reassessment should also be considered to confirm that no damage to the coronary artery has oc- 
curred.

\section{Conflict of Interest}

The authors declare that they have no competing interests related to this manuscript. The authors have no funding or financial relationships to disclose.

\section{References}

[1] Garg, N., Tewari, S., Kapoor, A., Gupta, D.K. and Sinha, N. (2000) Primary Congenital Anomalies of the Coronary Arteries: A Coronary: Arteriographic Study. International Journal of Cardiology, 74, 39-46. http://dx.doi.org/10.1016/S0167-5273(00)00243-6

[2] Angelini, P. (2007) Coronary Artery Anomalies: An Entity in Search of an Identity. Circulation, 115, 1296-1305.

[3] Lipton, M.J., Barry, W.H., Obrez, I., Silverman, J.F. and Wexler, L. (1979) Isolated Single Coronary Artery: Diagnosis, Angiographic Classification, and Clinical Significance. Radiology, 130, 39-47. http://dx.doi.org/10.1148/130.1.39

[4] Ogden, J.A. and Goodyear, A.V. (1970) Patterns of Distribution of the Single Coronary Artery. Yale Journal of Biology and Medicine, 43, 11-21.

[5] Vaishnava, P., Pyo, R., Filsoufi, F. and Sharma, S. (2011) Compression of an Anomalous Left Circumflex Artery after Aortic and Mitral Valve Replacement. The Annals of Thoracic Surgery, 92, 1887-1889. http://dx.doi.org/10.1016/j.athoracsur.2011.04.095

[6] Veinot, J.P., Acharya, V.C. and Bedard, P. (1998) Compression of Anomalous Circumflex Coronary Artery by a Prosthetic Valve Ring. The Annals of Thoracic Surgery, 66, 2093-2094. http://dx.doi.org/10.1016/S0003-4975(98)01082-0

[7] Castillo, J.G., Sanz, J., Fischer, G.W., Bowman, K. and Filsoufi, F. (2009) Management of Anomalous Left Circumflex Artery Encircling the Aortic Annulus in a Patient Undergoing Multivalvular Surgery. Journal of Cardiac Surgery, 24, 667-669. http://dx.doi.org/10.1111/j.1540-8191.2009.00930.x

[8] Yokoyama, S., Takagi, K., Mori, R. and Aoyagi, S. (2012) Aortic Valve Replacement in Patients with an Anomalous Left Circumflex Artery. Journal of Cardiac Surgery, 27, 174-177. http://dx.doi.org/10.1111/j.1540-8191.2011.01365.x 